\title{
Massive star-formation toward G28.87+0.07
}

\author{
J. J. Li ${ }^{1,2}$, L. Moscadelli ${ }^{2}$, R. Cesaroni ${ }^{2}$, R. S. Furuya ${ }^{3}$, Y. Xu ${ }^{1}$,
} T. Usuda ${ }^{3}$, K. M. Menten ${ }^{4}$ M. Pestalozzi ${ }^{5}$, D. Eliav ${ }^{5}$, and E. Schisano ${ }^{5}$

${ }^{1}$ Purple Mountain Observatory, Chinese Academy of Sciences, Nanjing 210008, email: jjli@pmo.ac.cn

${ }^{2}$ INAF-Osservatorio Astrofisico di Arcetri, Largo E. Fermi 5, I-50125 Firenze, Italy

${ }^{3}$ Subaru Telescope, National Astronomical Observatory of Japan, 650 North A'ohoku Place, Hilo, HI 96720, USA

${ }^{4}$ Max-Planck-Institut für Radioastronomie, Auf dem Hügel 69, 53121 Bonn, Germany

${ }^{5}$ INAF-Istituto Fisica Spazio Interplanetario, Via Fosso del Cavaliere 100, I-00133 Roma, Italy

\begin{abstract}
We investigated the high-mass star-forming region G28.87+0.07 by means of maser kinematics, including $\mathrm{H}_{2} \mathrm{O}, \mathrm{CH}_{3} \mathrm{OH}$, and $\mathrm{OH}$, and radio to infrared, continuum observations. All observational evidence suggests that these masers are associated with the same young star of 20-30 $\mathrm{M}_{\odot}$, still in the main accretion phase and surrounded by a rich stellar cluster.
\end{abstract}

Keywords. ISM: individual objects (G28.87+0.07) - ISM: kinematics and dynamics - masers techniques: interferometric

Understanding the process of high-mass star formation represents a challenge from both theoretical and observational points of view. A few years ago, we started an observational project to study the high-mass star-forming process by comparing interferometric images of thermal lines of molecular tracers with multi-epoch VLBI studies in three well known maser species $\left(\mathrm{OH}, \mathrm{CH}_{3} \mathrm{OH}\right.$ and $\left.\mathrm{H}_{2} \mathrm{O}\right)$. At present, three sources (IRAS 20126+4104, G16.59-0.05 and G23.01-0.41) have been already analyzed (Moscadelli et al. 2011, Sanna et al. 2010a, Sanna et al. 2010b). The results demonstrate that the synergy between VLBI, multi-species maser observations and (sub) mm interferometric observations of thermal molecular lines is crucial to achieve a multi-scale picture of the environment of newly formed massive (proto)stars. Here we represent our study of the high-mass star-forming region (HMSFR) G28.87+0.07.

We observed the HMSFR G28.87+0.07 with the VLBI phase-referencing technique in three powerful maser transitions: $22.2 \mathrm{GHz} \mathrm{H}_{2} \mathrm{O}$ (VLBA, four epochs), $6.7 \mathrm{GHz} \mathrm{CH}_{3} \mathrm{OH}$ (EVN, four epochs), and $1.665 \mathrm{GHz} \mathrm{OH}$ (VLBA, one epoch). In addition, we also performed VLA observations of the radio continuum emission at 1.3 and $3.6 \mathrm{~cm}$ with both the A- and C-arrays, and Subaru observations of the mid-infrared continuum emission. We also made use of data from the Hi-GAL/Herschel, ATLASGAL/APEX, as well as other surveys of the Galactic plane. More details on the observations are described by Li et al. 2012. From all these observations, we draw the following conclusions:

(a) The bipolar distribution of line-of-sight velocities and the general pattern of observed relative proper motions indicate that the water masers are tracing a (proto)stellar jet along the northeast-southwest direction (Fig. 1), which is also powering the large-scale outflow (Furuya et al. 2008).

(b) The positions of the $\mathrm{CH}_{3} \mathrm{OH}$ maser features roughly along the jet axis (Fig. 1), and the similarities in the spectral shape of the water and methanol maser emissions, suggest that also the $\mathrm{CH}_{3} \mathrm{OH}$ masers could originate in the same jet, albeit associated with more quiescent gas than that traced by the water masers. 


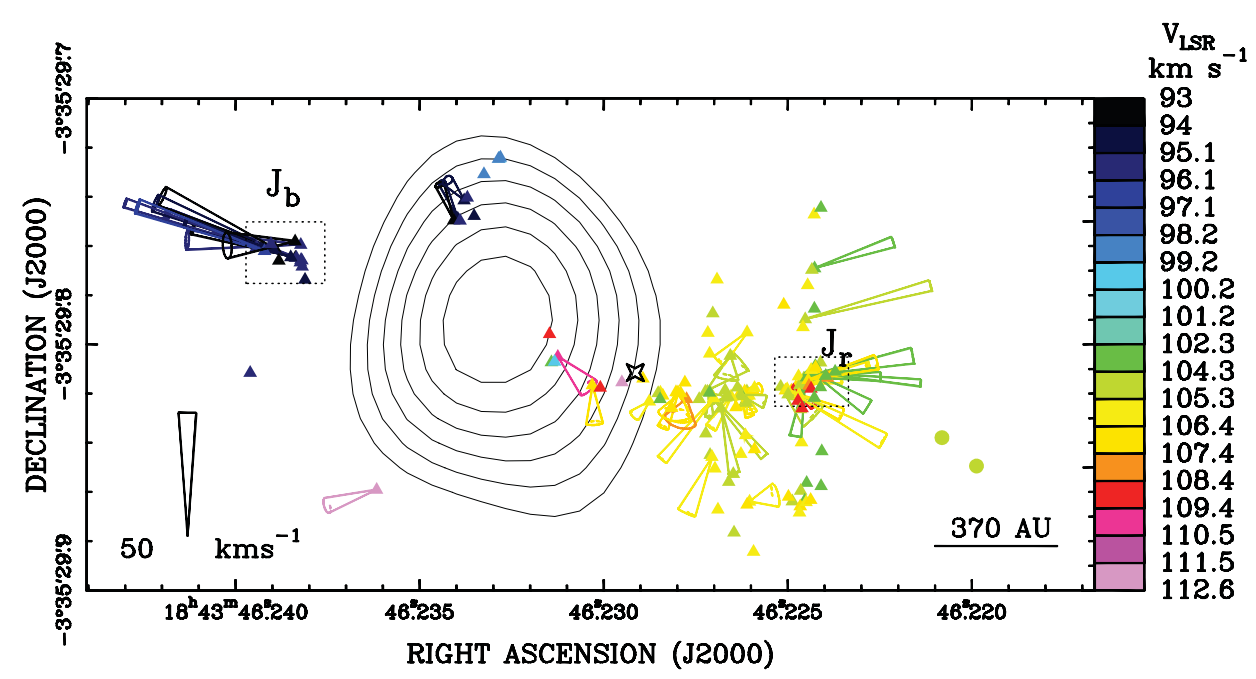

Figure 1. Proper motions of $22 \mathrm{GHz} \mathrm{H}_{2} \mathrm{O}$ masers (triangles) relative to their "center of motion" (marked with a cross), and spatial distribution of $6.7 \mathrm{GHz} \mathrm{CH}_{3} \mathrm{OH}$ masers (dots) in $\mathrm{G} 28.87+0.07$. Different colors are used to indicate the maser LSR velocities, according to the color scale on the righthand side of the plot. The cones indicate the $3-\mathrm{D}$ velocities of the water maser features relative to the "center of motion" . Points without an associated cone have been detected only over one or two epochs and the associated proper motion cannot be computed or is considered unreliable. The cone opening angle gives the $1 \sigma$ uncertainty on the proper motion direction. The length of the cone is proportional to the velocity, with the amplitude scale indicated in the lower left corner of the figure. Open dotted rectangles mark two clusters of water masers located at the northeast and southwest edge of the distribution (labeled " $\mathrm{J}_{b}$ " and " $\mathrm{J}_{r}$ ", respectively), moving fast and close to the northeast-southwest direction. The VLA $1.3 \mathrm{~cm}$ continuum emission is plotted with solid contours. Contour levels range from $40 \%$ to $90 \%$ of the peak emission $\left(0.62 \mathrm{mJy}\right.$ beam $\left.^{-1}\right)$ at multiples of $10 \%$.

(c) The continuum sources "HMC" is spatially associated with the observed $\mathrm{H}_{2} \mathrm{O}$, $\mathrm{CH}_{3} \mathrm{OH}$ and $\mathrm{OH}$ masers. Whether the free-free emission arising from this source is due to an HII region or a thermal ionized jet cannot be unambiguously established with the present data. However, we believe that the jet hypothesis is more consistent with the direction of the water maser motions, which outline expansion in a bipolar flow.

(d) The combined information obtained from IR observations, permits to estimate with good accuracy the luminosity $\left(2 \times 10^{5} L_{\odot}\right)$ and gas mass $\left(3 \times 10^{3} M_{\odot}\right)$ of the star forming region of $\mathrm{G} 28.87+0.07$, and establish that $\sim 90 \%$ of the luminosity is coming from the radio source "HMC". We conclude that this source must contain multiple stars, with the most massive being at least as luminous as $3 \times 10^{4} L_{\odot}$. The lack of an associated HII region indicates that the $20-30 M_{\odot}$ star must be still undergoing heavy accretion from the surrounding envelope.

This work was supported by the Chinese NSF through grants NSF 11133008, NSF 11073054, NSF 10621303, NSF 10733030 and the Key Laboratory for Radio Astronomy, CAS.

\section{References}

Furuya, R. S., Cesaroni, R., Takahashi, S., et al. 2008, ApJ, 673, 363

Li, J. J., Moscadelli, L., Cesaroni, R., et al. 2012, ApJ, accepted

Moscadelli, L., Cesaroni, R., Rioja, M. J., et al. 2011, A\&A, 526, 66

Sanna, A., Moscadelli, L., Cesaroni, R., et al. 2010a, A\&GA, 517, 71

Sanna, A., Moscadelli, L., Cesaroni, R., et al. 2010b, A\&A, 517, 78 